\title{
Online Monitoring of Three Dimensional Ledge Profile of Aluminum Electrolytic Cell
}

\author{
Zhengguang Xu, Liling Song*, Mingsheng Ju and Yu Xue \\ University of Science and Technology Beijing, China \\ *Corresponding author
}

\begin{abstract}
A ledge of certain thickness will be formed on the side wall of the cell during aluminum electrolysis process, its formation has a very important role for the normal production of aluminum electrolysis. First a three-dimensional unsteady heat transfer model with phase transformation is established by the finite difference method. Then the initial temperature field and the profile of initial ledge is calculated layer by layer and the nodes of ledge is corrected through iterative loop .Finally, the profile of the ledge will be simulated .It is found that the result calculated is good agreement with the test in cell .
\end{abstract}

Keywords-aluminum electrolytic cell; ledge profile; finite difference method; node modification

\section{INTRODUCTION}

In the practice of aluminum electrolytic production, the ledge has a outstanding effect on the current distribution, melt flow, current efficiency, energy consumption and cell life [1].

As early as in 1971, Haupi was the first to propose a onedimensional heat conduction model of calculating the ledge profile, which can be used to estimate the heat loss and the thickness of the ledge of the cell[2]. This one-dimensional heat transfer model is useful for calculating the local heat transfer loss of the electrolytic cell, but not for solving the ledge profile of the electrolytic cell and the error is too large. Therefore, the later research is based on the mathematical model of 2D or 3D. Chi Mei calculated the temperature distribution of the cathode of the electrolytic cell by using finite difference method, which provides a basis for the calculation of the ledge profile[3].

Considering the existence of phase change between the ledge and melt, H.A.Ahmed made some improvements in the study of model, therefore, they add the latent heat of phase change to the equation of mathematical control [4]. C. Bertrand established a quasi two-dimensional heat conduction model which described the heat conduction model containing the phase change process, and then use the iterative algorithm of conjugate gradient method to balance the contradiction between the amount of calculation and the calculation error [5].

This paper establishes a three-dimensional unsteady computational model which adds a factor of phase change for a aluminum electrolytic cell in Chongqing, and realize the online monitoring of the ledge profile through the $\mathrm{C}++$ programming.

\section{Three Dimensional Heat Transfer Model}

Figure $\mathrm{I}$ is the schematic diagram of aluminum electrolytic cell, the profile of ledge is defined by the ring groove shape of the layer of electrolyte crust.

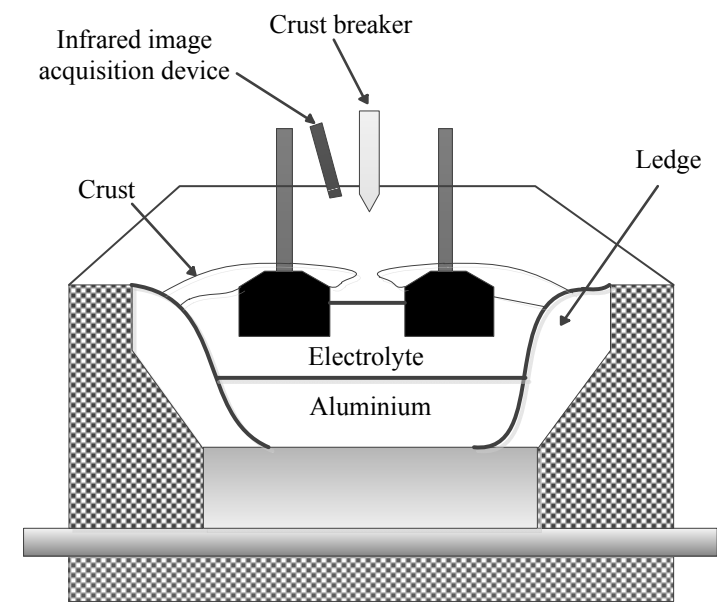

FIGURE I. ALUMINUM ELECTROLYTIC CELL

\section{A. Physical Model Simplification}

The side ledge of the electrolytic tank is only existed between the cathode and anode, so it is necessary to establish the heat transfer model of blue box in Figure II.

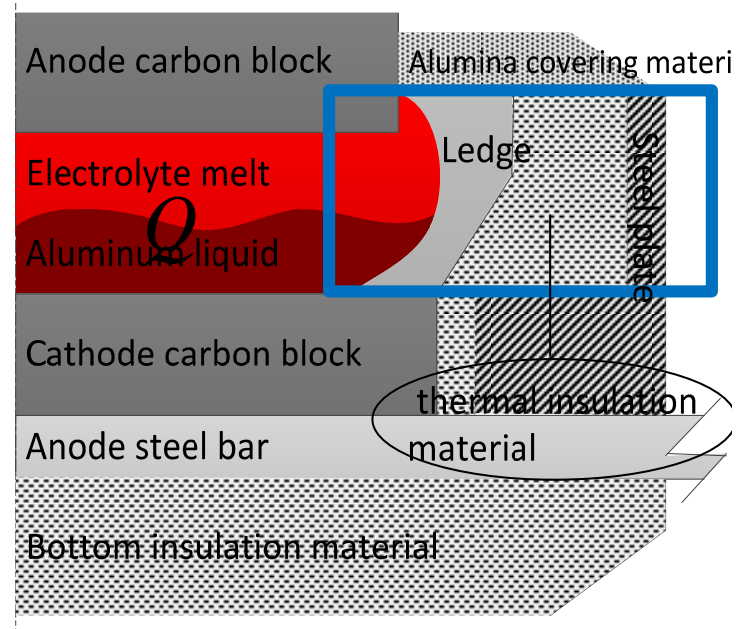

FIGURE II. SCHEMATIC DIAGRAM OF SIMPLIFIED MODEL

In this research area, we assume that the melting temperature is equal to the constant value, and the model is not required to be calculated by the model, so it is considered that the model in this research area does not contain internal heat source. And there is a mushy zone between the ledge and the 
melt because of the existence of the solid-liquid phase change. As time changes, the profile of the ledge is constantly changing, so the temperature field of the study area will change with time.

So the three-dimensional unsteady heat transfer model of the solid part and the mushy zone in this research area is:

$$
\rho c \frac{\partial T}{\partial t}=\frac{\partial}{\partial x}\left[k_{x} \frac{\partial T}{\partial x}\right]+\frac{\partial}{\partial y}\left[k_{y} \frac{\partial T}{\partial y}\right]+\frac{\partial}{\partial z}\left[k_{z} \frac{\partial T}{\partial z}\right]-\delta H \frac{\partial f}{\partial t}
$$

In the above formula, $\rho, \mathrm{c}$ refer to density and specific, $\mathrm{kx}, \mathrm{ky}$, $\mathrm{kz}$ is the thermal conductivity of the three directions.

The last term on the right-hand side of (1) accounts for the solid/liquid phase change. The enthalpy $\delta \mathrm{H}$ is defined as $\delta H=\rho \lambda, \lambda$ is the melting heat and the data are learned from [6], The liquid fraction $f$ varies as in the following manner[7]:

$$
f=f(T)=\left\{\begin{array}{cc}
o & T \leq T_{s} \\
\left(\frac{T-T_{s}}{T_{l}-T_{s}}\right) & T_{s}<T<T_{l} \\
1 & T \geq T_{l}
\end{array}\right.
$$

In the calculation of solid part, the liquid volume fraction is 0 , so the model hasn't the last item on the right side of (1), it's a typical three-dimensional unsteady heat transfer model.

The material in the area of phase change will release or absorb more heat in a relatively narrow temperature range. From the view of the surface, the phenomenon is equivalent to the material specific heat increases suddenly in the transformation process, and after the process of phase change it is restore the original state. Therefore, we can use the concept of equivalent specific heat to describe the phase change process of phase change material.

According (2), the volume fraction is determined by the temperature, so the change rate of the volume fraction can be expressed as $\frac{\partial f}{\partial t}=\frac{\partial f}{\partial T} \frac{\partial T}{\partial t}$, then the total heat transfer equation is:

$$
\rho\left(C+\lambda \frac{\partial f}{\partial T}\right) \frac{\partial T}{\partial t}=\frac{\partial}{\partial x}\left[k_{x} \frac{\partial T}{\partial x}\right]+\frac{\partial}{\partial y}\left[k_{y} \frac{\partial T}{\partial y}\right]+\frac{\partial}{\partial z}\left[k_{z} \frac{\partial T}{\partial z}\right]
$$

\section{B. Boundary Condition}

A complete mathematical description of the specific heat conduction problem, in addition to heat conduction differential equations, but also give the corresponding conditions for the definite solution.

Therefore, we conducted a survey on the cell structure and the size of Chongqing aluminum electrolytic plant, and gives the study area boundary conditions:
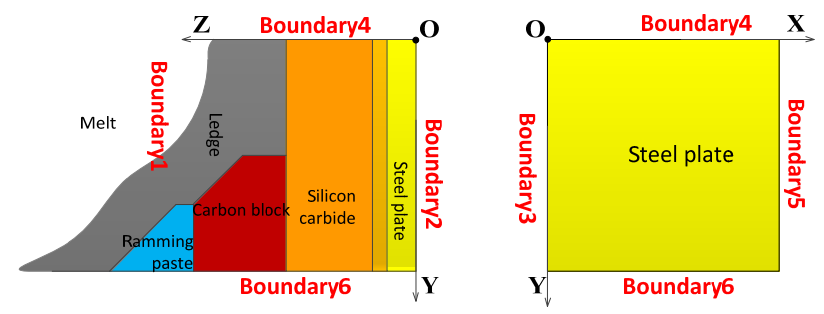

FIGURE III. BOUNDARY OF COMPUTATIONAL REGION

- The electrolyte and the aluminum liquid flow violently in the aluminum electrolytic tank and their heat exchange is sufficient, therefore, the temperature of the contact part between the melt and the ledge can be considered as the same.

- The surface of the steel plate at the boundary 2 needs to be measured by the thermocouple, and the temperature distribution of the whole surface is fitted by interpolation method, which is the first kind of boundary condition.

- Steel at boundary 2 will produce heat exchange with surrounding in the form of heat convection. The coefficient of heat transfer $\mathrm{h} f$ between the steel and surrounding air is $\left(\mathrm{T}_{\mathrm{s}}\right.$ is average temperature of steel plate, $T_{b}$ is temperature of surrounding, $\sigma_{0}$ is the StefanBoltzmann constant $\left(5.667 \times 10^{-8} \mathrm{~W} \cdot \mathrm{K}\right), \quad \varepsilon \quad$ is the blackness of steel, it take 0.96 generally):

$$
h_{f}=h_{0}+\sigma_{0} \varepsilon \frac{\left[\left(T_{s}+273.15\right)^{4}-\left(T_{b}+273.15\right)^{4}\right]}{\left(T_{s}-T_{b}\right)}
$$

- For "boundary 3" and "boundary 5", this paper only studies the part of the middle part of the cell, so the boundary 3 and the boundary 5 are given as the open boundary, and the boundary conditions are not loaded.

- Similarly to the "boundary 4" and "boundary 6", the structure and material of their position are very complicated, and the boundary condition is not loaded.

\section{Heat Transfer Model Based on Finite Difference}

Numerical method is a kind of approximation method with sufficient accuracy, of which the finite difference method is the most widely used method, especially the popularity of the electronic computer, this method has been widely used.

\section{A. Grid Division}

In fact, the grid division is that making the solution area discrete. Figure IV is a reduced graph of the solution area. 


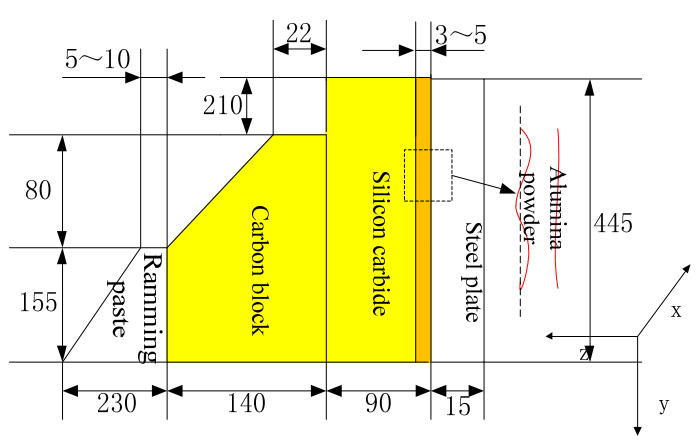

FIGURE IV. REDUCED GRAPH OF THE SOLUTION AREA

In order to ensure that there have enough number of nodes to meet the accuracy of discretization after grid division, so we can set all the grid step in about $5 \mathrm{~mm}$.

When crossing the medium, the node lies squarely in the interface of medium. The calculated results when the nodes in the interface of medium can represent the local temperature distribution of interface more accurately than the nodes not. The stepped grid can instead of inclined plane of the artificial ramming paste and irregular carbon block, as shown in figure V.

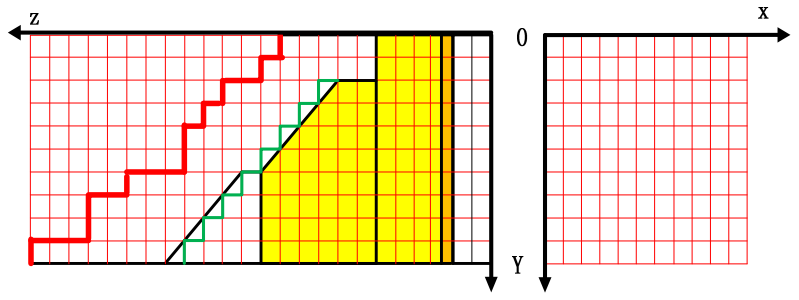

FIGURE V. SKETCH MAP OF GRID DISTRIBUTION

\section{B. Discretization of Heat Transfer Model}

We can use the backward difference for the left side of the (3) and use the central difference for the right side of the (3) to get a three dimensional unsteady heat conduction difference equation with constant physical property and no internal heat source.

$$
\begin{aligned}
\rho\left(C+\lambda \frac{\partial f}{\partial T}\right) \frac{T_{i, j, k}^{p}-T_{i, j, k}^{p-1}}{\Delta t} & =k_{x 1} \frac{T_{i+1, j, k}^{p}-T_{i, j, k}^{p}}{\Delta x^{2}}+k_{x 2} \frac{T_{i-1, j, k}^{p}-T_{i, j, k}^{p}}{\Delta x^{2}}+k_{y 1} \frac{T_{i, j+1, k}^{p}-T_{i, j, k}^{p}}{\Delta y^{2}} \\
& +k_{y 2} \frac{T_{i, j-1, k}^{p}-T_{i, j, k}^{p}}{\Delta y^{2}}+k_{z 1} \frac{T_{i, j, k+1}^{p}-T_{i, j, k}^{p}}{\Delta z^{2}}+k_{z 2} \frac{T_{i, j, k-1}^{p}-T_{i, j, k}^{p}}{\Delta z^{2}}
\end{aligned}
$$

For the boundary condition of the heat transfer model, it only need to make the the third kinds of boundary conditions on the steel plate discretized.

$$
-k\left(\frac{T_{i, j, 1}^{p}-T_{i, j, 2}^{p}}{\Delta z}\right)=h_{f}\left(T_{i, j, 1}^{p}-T_{b}\right)
$$

In the above formula, $\mathrm{k}$ is thermal conductivity of steel plate, $T_{i, j, 1}^{p}$ is the temperature value of first layer nodes at $\mathrm{P}$ time,
$T_{i, j, 2}^{p}$ is the temperature value of second layer nodes at $\mathrm{P}$ time, $\mathrm{T}_{\mathrm{b}}$ is the ambient temperature.

\section{Special Node}

In heat transfer process, in addition to the heat conduction in the same material, there are a number of nodes in special positions, they can not be handled according to the general situation.

\section{1) Node at the interface}

According to the location of the node, the node relationship can be divided into six types of relationship shown in Figure VII.

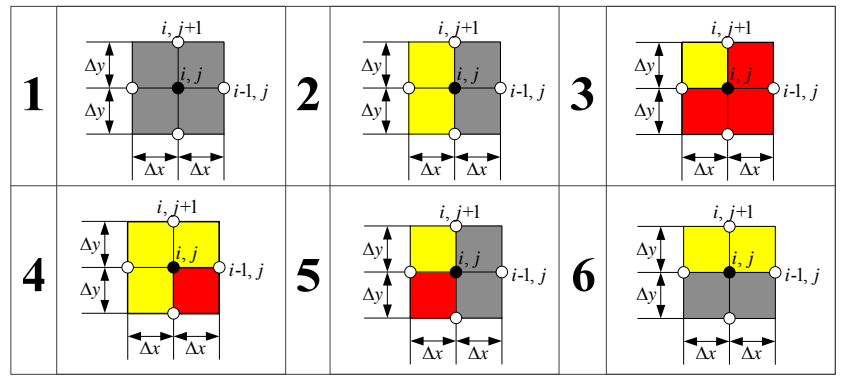

FIGURE VI. NODE RELATIONSHIP

The nodes temperature of different locations are shown in figure 6 is still used the (5) to calculate. In addition to the first node type, the equivalent heat transfer coefficient between other nodes is calculate using the method of heat balance, thus, the heat conduction can be better described at the interface.

\section{2) Node in mushy zone}

The mushy zone between the ledge and the melt is the transition zone between solid and liquid, which can be seen as the different state of the same kind of medium.

In this paper, the crystallization point is used as the point on the ledge. After getting the temperature of each layer, we can check the crystallization point and then mark it. Generally, We think that the width of mushy zone is about $5-10 \mathrm{~mm}$, which is one to two grid.

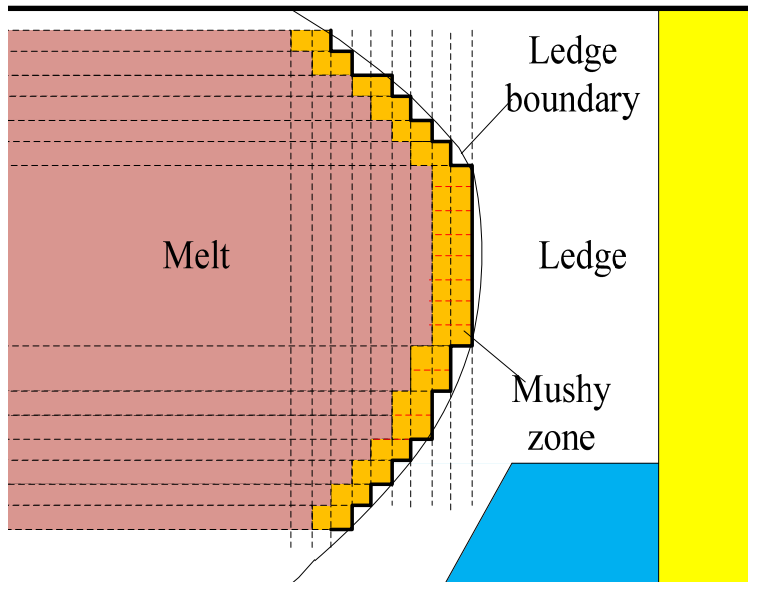

FIGURE VII. LOCAL SKETCH MAP OF MUSHY ZONE 
As shown in figure VII, we assume that the two nodes in the back of the crystallization point are in mushy zone and the nodes in the back of mushy zone are in melt of which temperature is constant.

We can calculate the temperature of the node in the mushy according to the above model, but the equivalent thermal conductivity in the model need to be calculated by the harmonic mean method.

\section{Simulation PROCESS}

\section{A. Basic Steps of Simulation Program}

1) First: We need to establish a three-dimensional heat transfer model and input the size of the cell and various parameters.

2) Second: The temperature field between the steel plate and the ledge and the initial ledge profile are obtained by loading boundary conditions.

3) Third: The difference between the calculated surface temperature and the actual temperature of the steel plate can be calculated on the basis of the above results, and determine whether the difference is within the error range.

4) Fourth: If not, we can adjust the nodes of the ledge and establish a new ledge profile,then repeat 3), 4); else run 5)directly.

5) Finally: we can obtain the final ledge profile and the temperature field.

\section{B. Mobile Node Strategy}

In the calculation process, there exist deviations in the method of choosing parameters or approximate treatment in the course of the recursion, and considering the variety of the ledge profile, we need to make point to point correction for the profile of the initial ledge.

Nodes movement only to change the coordinates of the $\mathrm{Z}$ direction. In addition, we set a special mobile step $L_{\text {STEP }}$. For the difference between the actual surface temperature and the surface temperature of reverse sliding, according to the extent of the difference to determine the size of the mobile. The following node mobility strategy is adopted:

$$
X^{k}=X^{k-1}+L_{\text {STEP }} \frac{T_{b}-T_{s}}{T_{0}-T_{e}}
$$

In the above formula, $X^{k}, X^{k-1}$ is the coordinates of the ledge node after the $\mathrm{k}$ and $\mathrm{k}-1$ iterations, $T_{b}, T_{s}$ is the surface temperature of reverse sliding and the actual surface temperature, $T_{e}$ is the initial crystal temperature, $T_{0}$ is the melt temperature. The formula shows that the error between the surface temperature calculated and the actual temperature of the steel plate can adjust the position of the ledge.

Cutoff condition: the moving distance between two iteration is in a certain range that we set.

\section{CAlculation Result}

In order to verify the rationality and accuracy of the crust estimate algorithm, this paper used the steel temperature measured directly as the boundary condition to carry out the numerical simulation. The simulation result of threedimensional profile of the ledge is shown in the figure VIII, the temperature field in the side of the aluminum cell $\mathrm{ZY}$ section is shown in figure IX.

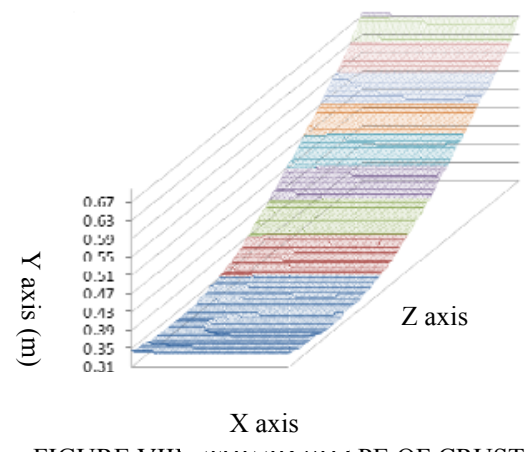

FIGURE VIII. THE 3D SHAPE OF CRUST

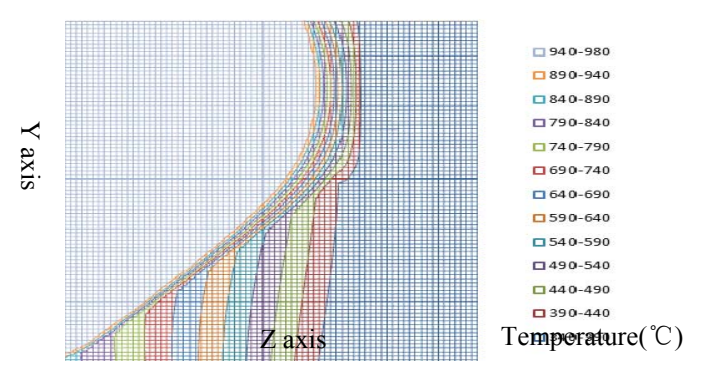

FIGURE IX. TEMPERATURE FIELD DISTRIBUTION OF ZY CROSS SECTION

The 3D ledge profile and temperature distribution show that tank lining materials in aluminum tank has a very good effect of heat insulation, the temperature gradient in the thermal insulation material is not large and it'd good for the insulation effect; as well as, it showed that the minimum distance between the ledge and lateral steel plate is about in the vicinity of the interface of molten electrolyte and aluminum liquid. as we see from the section, the ledge profile is parabolic shape which extend naturally along the irregular carbon block edge .

The results of the simulation show that there is insufficient and the thickness of the ledge on the inclined plane is very thin which illustrate the electrolytic cell is overheating, electrolyte melt may have corroded groove lining material. On the whole, the general trend of simulation results accord with the actual conditions of the crust shape and the result calculated is good agreement with the test in cell .

\section{CONCLUSION}

This paper analyzes the heat transfer phenomenon of the temperature field from the outer wall to the side part of the ledge in the aluminum electrolysis cell, and the phenomenon is accurately simulated by the numerical method, the result calculated is good agreement with the test in cell . 
The study on ledge profile can provide the accurate heat transfer model for the calculation of thermal equilibrium of electrolytic ell, and carried out the simulation which can monitor the time-varying ledge profile, it provide the basis for the correction of electrolytic cell lining materials and internal measurement.

\section{REFERENCES}

[1] Haishi Liu, "The tank hearth of large scale prebaked aluminum electrolytic cell," Light metal, 1994, (7): 34-36.

[2] W. E. Haupin, "Calculating Thickness of Containing Walls Frozen from Melt," TMS-AIME Annual Meeting Pager, New York, 1971, pp. 305309.

[3] Chi Mei, Wang You, Qianpu Wang, Hubin Tu, "Research and development of the simulation software for the online display of the groove of the aluminum reduction cell, " Department of Applied Physics and Heat Engineering, Central South University of Technology, Changsha, April 1997, vol. 28, pp.138-141.

[4] HA Ahmed, FA Elrefale, MF El-Demerdash, SM El-Raghy, Z Bassuny, "Development of a Thermal Model for prebaked Aluminum Reduction at the Aluminum Company of Egypt, " Light Metals, 1993, pp.258-266.

[5] Bertrand, Marois, Désilets, Soucy, Lacroix, “ A combined 2D inverse predictions and experimental analysis for the bank formation inside a metallurgical reactor," International Journal of Heat and Mass Transfer, 2013, 59(1), pp.58-65.

[6] M. LeBreux , M. Désilets, M. Lacroix, “Fast inverse prediction of phase change banks in high temperature furnaces with a Kalman filter coupled with a recursive least-square estimator , "Int. J of Heat \& Mass Trans , $2010,53(23-24)$, pp.5250-5260.

[7] V. R. Voller, C. R. Swaminthan , "General Source-based Method for Solidification Phase Change, " Numerical Heat Transfer, 1991, 19(2), pp. 175-189. 